\section{COMUNICACIÓN EN LA INVESTIGACIÓN Y SU RELACIÓN CON LA SATISFACCIÓN LABORAL DEL PERSONAL ACADÉMICO INVESTIGADOR E INFRAESTRUCTURAS UNIVERSITARIAS}

\author{
Lamberto Luis Benito del Valle \\ Universidad del País Vasco \\ lamberto.benitodelvalle@ehu.es \\ Galder Guenaga Garay \\ Universidad del País Vasco \\ galder.guenaga@ehu.es \\ Xabier Renteria-Uriarte \\ Universidad del País Vasco \\ xabier.renteria@ehu.es \\ María Jesús Luengo-Valderrey \\ Universidad del País Vasco \\ mariajesus.luengo@ehu.es
}

Cómo citar este artículo/Citation: Benito del Valle, L. L., Guenaga Garay, G., Renteria-Uriarte, X. y Luengo-Valderrey, M. J. (2016). Comunicación en la investigación y su relación con la satisfacción laboral del personal académico investigador e infraestructuras universitarias. Arbor, 192 (780): a339. doi: http://dx.doi.org/10.3989/arbor.2016.780n4015

Recibido: 18 julio 2014. Aceptado: 30 junio 2016.

RESUMEN: La comunicación de resultados de investigación resulta preeminente como criterio de calidad e indicador de posicionamiento en rankings mundiales de universidades. Asimismo, se asume que el rendimiento laboral se ve influenciado por el grado de satisfacción de los miembros de la organización con su ambiente laboral y su actividad. La aplicación del modelo estadístico de ecuaciones estructurales a una encuesta sobre satisfacción, motivación e implicación laboral realizada al personal académico investigador de la UPV/EHU concluye que dicha comunicación es potenciada y explicada (en orden descendente) por la posibilidad de: trabajar en ambientes personales no universitarios bien equipados; desarrollar habilidades y plantearse nuevos retos, formarse y proveerse de instrumentos materiales e inmateriales; incentivos, reconocimiento y agilidad burocrática; percepción personal del rendimiento productivo derivado de la satisfacción laboral; satisfacción personal en tareas de comunicación en congresos y otros escenarios; y satisfacción en tareas de preparación y redacción de material de investigación.

PALABRAS CLAVE: Análisis cuantitativo; calidad; comunicación científica; contexto universitario; investigación; motivación; revistas; satisfacción.

\section{REPORTING RESEARCH RESULTS AND ITS RELATION WITH ACADEMIC RESEARCHER'S JOB SATISFACTION AND WORKING FACILITIES}

Copyright: ( 2016 CSIC. Este es un artículo de acceso abierto distribuido bajo los términos de la licencia Creative Commons Attribution (CC BY) España 3.0.

ABSTRACT:The communication of research results is preeminent as a quality criterion and position indicator, prefixing even major world university rankings. At present, it is assumed that job performance is influenced by the degree of satisfaction of the members of an organization with their work environment and activity. Based on a survey of job satisfaction, motivation and involvement conducted among academics from the UPV/EHU, and through the application of structural equation modeling, this paper concludes that this communication is enhanced and explained (in descending order) by: the possibility of working in non-university personal but well-equipped rooms; the opportunity to develop skills and set new challenges as well as to receive more training and other tangible or intangible instruments; incentives, recognition and bureaucratic agility; personal perception of productive efficiency due to job satisfaction; personal satisfaction in communication tasks at conferences and other venues; and satisfaction obtained by preparing and writing research material.

KEYWORDS: Quantitative analysis; quality; scientific communication; scholarly context; research; motivation; journals; satisfaction. 


\section{INTRODUCCIÓN Y ESTADO DE LA CUESTIÓN}

La comunicación de los resultados de la investigación es un factor de vital y creciente importancia para el desarrollo humano y la innovación tecnológica. En la actualidad se considera que no hay investigación científica sin publicar los resultados para compartirlos y discutirlos con la comunidad científica: "[r]esearch is complete only when the results are shared with the scientific community" (APA, 2010, p. 9) o, más sintéticamente, "[r]esearch is not complete until it is published" (Morris, 1998, p. 256). El nuevo informe de la National Science Foundation de EEUU (2014) sobre indicadores de ciencia y de ingeniería vuelve a otorgar especial relevancia a los artículos científicos y a los criterios referidos a ellos (por ejemplo, y en especial, la coautoría internacional). Pero ¿de qué tipo de publicación estamos hablando? El medio preferido son las revistas científicas y actas de conferencias, pero lo más valorado no es ya 'publicar', sino publicar en forma de 'artículos indexados'. Se entiende por tales a los publicados en revistas de renombre que se indexan en bases de datos mundialmente reconocidas, tales como la Web of Science o Scopus, y su impacto se mide según la frecuencia de citas en otros trabajos posteriores.

En el caso de las universidades, la investigación conforma, junto con la docencia, una de sus dos funciones tradicionales. No obstante, la investigación es actualmente preeminente como factor de calidad e indicador de posicionamiento. Los principales rankings mundiales de universidades, como Times Higher World University Ranking (THWU), Academic Ranking of World Universities (ARWU, más conocido como Shanghai Ranking), y QS World University Ranking (QSWU), se basan en gran medida en este criterio. Por ejemplo, en el THWU, el 65\% del ratio corresponde a criterios de investigación sin mayor matiz, el 23\% corresponde a esos criterios como medida de la calidad docente, y solo un 4,5\% implica un criterio de docencia sin relación con la investigación (World University Rankings 2011-12 methodology).

Dentro de dicha investigación universitaria, la comunicación tiene una importancia capital. Todos los criterios de investigación de los rankings de universidades, incluyendo los ingresos, la reputación, los premios, etc., vienen determinados por los 'artículos indexados'. Así, si bien el personal docente e investigador universitario utiliza para la comunicación de sus investigaciones diversos medios y soportes (artículos y libros, artículos divulgativos y de opinión en mass media o web, e incluso inserciones en los apuntes para el alumnado), los rankings dejan claro el criterio principal: 'artículos indexados' y 'citas' están presentes en los tres rankings mencionados (en el THWU, con un $8,5 \%$ y un $30 \%$; en el ARWU, con $40 \%$ y $20 \%$; y en el QSWU las citas con un $20 \%$ ), y determinan el resto de criterios de investigación por esa importancia actual ya apuntada.

Por otra parte, el llamado Espacio Europeo de Educación Superior (EEES), iniciado con las Declaraciones de la Sorbona (1998) y de Bolonia (1999), ha potenciado el aprendizaje participativo con Proyectos de Innovación Educativa o PIE. Se trata de programas de 'investigación-en-acción', es decir, la innovación docente se implanta y testa en el aula con criterios de producto científico, y al final existe el compromiso de comunicar los resultados. Por ello, resultan apropiados para entender de forma más extensa los determinantes de la comunicación en la investigación universitaria. La innovación docente universitaria y sus formas concretas en PIE han ido analizándose a modo de 'zoom': de reflexiones analíticas (House, 1988) y estudios generales (de León, 2002; Quintanal Díaz, 2010; Herrera, 2010) se ha ido pasando a sus concreciones experimentales en aulas (García, 1996; García, Ruíz, y Noche, 2010), y el análisis cuantitativo que aquí se presenta los considera como variable de comunicación de la investigación universitaria.

La comunicación de los resultados de investigación se estudia desde hace décadas (Mann y Likert, 1952), pero ha experimentado un importante auge en la moderna era de la tecnología digital (Barjak, 2006; Cohen, 1996; Kling y McKim, 2000; Odlyzko, 2002). Un buen indicador del desarrollo del área es la propuesta de meta-modelos explicativos (Björk, 2007; Shaw, 1981), para el que se prevé un gran dinamismo futuro (Beall, 2014; Rupp-Serrano y Antell, 2013; Shorley y Jubb, 2013) y una mayor democratización en los accesos (Solomon, 2013). Sin embargo, a pesar de todo, el campo no se halla exento de algunos peligros, y por ello debe mantenerse ágil y creativo incluso a nivel de políticas microespecíficas (Harley, 2013; Phillips, 2011). En este escenario, el presente trabajo pretende concretar los determinantes de esta comunicación de resultados de investigación, en la esfera universitaria, con referencia a estas dos variables de tanta relevancia: los artículos indexados y los PIEs, puesto que (sobre todo en el primer caso) son los factores tenidos en cuenta por los rankings mundiales de universidades y porque, además, sin la publicación previa no se puede acceder a otros factores motivadores de la investigación, como son los incentivos económicos (sexenios) y académicos (acreditaciones). 
La determinación de esta comunicación de la investigación tiene como contexto ineludible a la satisfacción y bienestar laboral experimentados por el trabajador con la actividad propia y con su contexto organizacional. La moderna -y profusa- literatura de gestión ha evidenciado los determinantes y condicionantes de la satisfacción laboral desde sus inicios (empezando por los experimentos de Elton Mayo, 1933; teorías X e Y de McGregor, 1957; teoría Z de Ouchi, 1981; teoría de los dos factores de Herzberg, 1968) hasta el discurso de los gurús actuales del management (Drucker, 1993; Hackman y Oldham, 2005; Peters y Waterman, 1983; Pfeffer, 1994; Saratxaga, 2007; Senge, Roberts, Ross, Smith y Kleiner, 1995; Tapscott y Williams, 2007). La aproximación a esta dimensión se ofrece aquí mediante los factores infraestructurales de carácter físico (instrumentos, espacios y otras infraestructuras) e inmaterial (incentivos, reconocimiento, etc.), los de rendimiento productivo, y los factores de índole emocional y mental del personal investigador, según las propias percepciones y la satisfacción laboral subjetiva derivada por parte de los sujetos en estudio.

En lo que respecta a la satisfacción laboral, existen diversas maneras de cuantificarla y modelarla (Bussing, Bissels, Fuchs y Perrar, 1999; Conklin y Desselle, 2007), como es de esperar dada la evolución teórica que la considera un importante factor de rendimiento productivo (Elçi y Alpkan, 2009; Falkenburg y Schyns, 2007; Judge, Thoresen, Bono y Patton, 2001; Koyuncu, Burke y Fiksenbaum, 2006; Maharaj y Schlechter, 2007). En el ámbito universitario las aplicaciones tienden también a una casuística amplia (Burke, Koyuncu y Fiksenbaum, 2008; Conklin y Desselle, 2007; Koyuncu, Burke y Fiksenbaum, 2006; Pearson y Moomaw, 2005; Smith, 2009). El presente estudio opta por el criterio de 'satisfacción por absorción en la tarea', propia de la vivencia de 'experiencia óptima', 'flujo' o flow (Csíkszentmihályi, 1975; Csíkszentmihályi, 1990; Nakamura y Csíkszentmihályi, 2012). Se trata de que los sujetos en estudio consideren estas 'experiencias óptimas por absorción de la atención', es decir, los momentos y períodos de actividad productiva en que la mente se halla completamente concentrada, absorta y unida a dicha actividad, y que esta literatura ha mostrado como uno de los determinantes más importantes de la satisfacción y el rendimiento laborales. Adicionalmente, se suaviza el sesgo de la medición subjetiva de la propia satisfacción al ser fácilmente localizable por el sujeto, quien carece de motivación para valorarla al alza al no tener, al menos todavía, connotaciones sociales como otras preguntas más conocidas y asumidas.
Una variable frecuente en la literatura descrita es la satisfacción con la vida de los sujetos, existiendo también una amplia literatura enfocada en la esencia de la propia variable (usualmente conocida como Subjective Well-being, veáse principalmente Diener, 2009; Kahneman y Krueger, 2006; Eid y Larsen, 2008). Y en relación al efecto de las variables de infraestructura física sobre el rendimiento, se mantiene aquí el criterio correspondiente de estudiar su efecto en variables de bienestar y satisfacción, una vez la inversión en capital físico previo se acepta como dada y externa al modelo (Aitken, 1982; Castelli, Manghi y Thanos, 2013; Elçi y Alpkan, 2009; Røssberg, Eiring y Friis, 2004; Spence Laschinger, Finegan, Shamian y Wilk, 2004).

En suma, el objetivo de este estudio es analizar los determinantes de la comunicación de los resultados de investigación en una época en la que la 'comunicación indexada' de la investigación ocupa un lugar central en las preocupaciones socioeconómicas de innovación y desarrollo y, sobre todo, en las universidades, al resultar -vía rankings- un factor condicionante de sus resultados económicos por el efecto llamada en los clientes (futuros alumnos, organizaciones colaboradoras...) y por su probable efecto en otras vías de financiación (subvenciones estatales, organizaciones partners...). El estudio toma como base los valores e interrelaciones de esas variables dentro del colectivo de personal docente e investigador de la Universidad del País Vasco (UPV/EHU), con resultados presumiblemente extrapolables y de interés para investigadores y órganos de gestión, a fin de enfocar mejor las decisiones personales y colectivas al respecto.

\section{MATERIAL Y MÉTODOS}

Este estudio trata de valorar el nivel de satisfacción, motivación e implicación de los miembros de la UPV/ EHU con su Universidad, así como los factores y aspectos asociados a dichos niveles.

Para ello, el presente trabajo toma como punto de partida las respuestas del profesorado y su vínculo con el número de artículos indexados y PIEs en los que han participado.

\subsection{Base de datos y muestra}

La base de datos primaria son los resultados de una encuesta sobre satisfacción, motivación e implicación laboral realizada, previo consentimiento informado, al personal docente e investigador de la UPV/EHU durante febrero de 2014. Dicha encuesta mide la satisfacción del personal docente e investi- 
gador en base al modelo Flow State Scale (Jackson, 1996; Jackson y Eklund, 2002), adaptada al universo al que se dirige y ampliada con cuestiones sobre gestión y resultados que pueden influir en su nivel de satisfacción, motivación e implicación. Ha sido testada y validada, mediante una revisión de 100 expertos de la UPV/EHU tras la que se han incorporado las sugerencias recibidas. A continuación, se ha procedido a testar ambos prototipos con otros 100 expertos de la UPV/EHU, tras lo que se ha establecido la encuesta definitiva. A su vez, se ha realizado el alfa de Cronbach de las propuestas inicial y definitiva para conocer su grado de fiabilidad. Ambas ofrecen resultados superiores a 0,8 , siendo mejores los de la propuesta definitiva que es la que se decide utilizar. Por lo tanto, se puede decir que la encuesta pasada cumple con las premisas de validez y fiabilidad.

Se ha recogido la opinión de 1.052 profesoras/es, sobre un total de 5.676, lo que dota a la muestra de un nivel de confianza del $99 \%$, con un margen de error del 3,58\%. Esto permite la aplicación del modelo SEM (ecuaciones estructurales) con garantías (Ruiz, Pardo y San Martín, 2010). El 46,77\% pertenecen al área de ciencias humanas y sociales, mientras que el $52,23 \%$ restante se encuadran en la ciencias experimentales y tecnológicas, por lo que los resultados representan el sentir del colectivo en su conjunto.

\subsection{Descripción de las variables}

Como variable que sintetiza actualmente la comunicación de los resultados de investigación en la Universidad, el sistema de 'artículos indexados' genera interés y tensión extra (el objetivo ya no es únicamente publicar para transferir conocimiento, sino publicar en revistas de alto impacto) para el investigador (Neuman, Davidson, Joo, Park y Williams, 2008), pero se obtienen ventajas de medición a nivel global (rankings internacionales) y particular (encuestas subjetivas). La variable al caso en este estudio es el Número de artículos indexados (PUBL), según la información aportada por el propio investigador. Esta variable de artículos indexados, que refleja normalmente investigaciones no docentes, se complementa con la participación en PIEs o programas formales de investigación y comunicación docente.

Para analizar los determinantes de la comunicación en la investigación en el sentido de la moderna literatura de gestión, se ponen estas variables (PUBL y PIE) en relación con variables de satisfacción que van de nada a totalmente satisfecho. Concretamente, se considera la propia variable de satisfacción con el trabajo (TP1) enmarcada con la satisfacción con la vida (TP2) y concretada en las vivencias de 'experiencia óptima' o 'absorción eficiente en la tarea' (TF3, TF4, TF5, TI3, TI4, TI5) en términos de frecuencia (TF de diaria a anual) e intensidad (TI de muy a nada intensa), y con la apreciación subjetiva de su efecto en el rendimiento laboral (TR1) y en la calidad del trabajo (TR2). Se las denominará 'variables satisfacción'.

$\mathrm{Y}$, finalmente, se estudia el efecto de las variables de infraestructura en este contexto, en función de la percepción subjetiva de su idoneidad: herramientas de formación (UF2), instrumentos (UF3), espacios y su equipamiento (UE1 y UE3), además de infraestructuras más inmateriales como los incentivos (UA1), el reconocimiento (UA2), o la agilidad burocrática (UA3). $\mathrm{Y}$, además, se contrasta el rol de los espacios personales no universitarios (UE2) o la potenciación percibida de habilidades y retos personales gracias a las infraestructuras (UF1). Se las denominará a todas ellas 'variables facilitadoras' y van de nada a totalmente facilitadoras. Todas estas variables se miden con una escala Likert de 7 tramos en la que el valor 1 es el mínimo y el 7 es el máximo.

Un resumen de estas variables se presenta en la Tabla I.

\subsection{Tipo de análisis estadístico}

El objetivo del estudio es establecer relaciones causales entre la satisfacción y la comunicación de resultados de investigación, para lo que se valdrá de modelos SEM que estiman parámetros estructurales y proporcionan información completa sobre la relación causa-efecto teórica (Bisquerra Alzina, 1989), confirmando dichas relaciones mediante el análisis de muestras (Jöreskorg, 1979).

La utilización del paquete estadístico SPSS con su módulo AMOS ha permitido (Arbuckle, 2006):

- Realizar el análisis descriptivo para conocer su valor medio y su normalidad uni y multivariante.

- Aplicar el análisis factorial de componentes principales (ACP) para extraer los constructos subyacentes que conforman el modelo de medida estructural.

- Comprobar la idoneidad de los indicadores que los explican para obtener el modelo definitivo de medida.

- Examinar la relación causal de dependencia e interdependencia entre los constructos. 
Tabla I. Variables incluidas en el estudio

\begin{tabular}{|c|c|c|}
\hline Variables Satisfacción & Variables Facilitadoras & $\begin{array}{c}\text { Variables Comunicación de Resultados } \\
\text { de Investigación }\end{array}$ \\
\hline $\begin{array}{l}\text { TF3-Frecuencia preparando alguna } \\
\text { investigación (en laboratorios, consul- } \\
\text { tando bases de datos, en bibliotecas...) }\end{array}$ & $\begin{array}{l}\text { UF1-Me permite utilizar y desarrollar mis } \\
\text { habilidades y plantearme nuevos retos } \\
\text { UF2-Me provee de herramientas de for- }\end{array}$ & $\begin{array}{l}\text { PIE-No proyectos innovación educativa } \\
\text { PUBL-№ publicaciones en revistas indexadas }\end{array}$ \\
\hline $\begin{array}{l}\text { TF4-Frecuencia redactando material } \\
\text { de investigación }\end{array}$ & $\begin{array}{l}\text { mación para mejorar mi capacitación } \\
\text { UF3-Me provee de instrumentos materiales }\end{array}$ & \\
\hline $\begin{array}{l}\text { TF5-Frecuencia participando activa- } \\
\text { mente en congresos, seminarios,... }\end{array}$ & $\begin{array}{l}\text { e inmateriales para realizar mis actividades } \\
\text { UE1-En los espacios que me adjudica la }\end{array}$ & \\
\hline $\begin{array}{l}\text { TI3-Intensidad preparando alguna in- } \\
\text { vestigación (en laboratorios, consul- } \\
\text { tando bases de datos, en bibliotecas...) }\end{array}$ & $\begin{array}{l}\text { Universidad, aunque sean compartidos } \\
\text { UE2-En un ambiente personal, como mi } \\
\text { casa u otro espacio, que sienta como propio, }\end{array}$ & \\
\hline $\begin{array}{l}\text { TI4-Intensidad redactando material } \\
\text { de investigación } \\
\text { TI5- Intensidad participando activa- } \\
\text { mente en congresos, seminarios,... }\end{array}$ & $\begin{array}{l}\text { aunque no esté suficientemente equipado } \\
\text { UE3-Dentro de la UPV/EHU, en un es- } \\
\text { pacio suficientemente equipado que no } \\
\text { tenga que compartir }\end{array}$ & \\
\hline $\begin{array}{l}\text { TR1-Mi rendimiento laboral aumenta } \\
\text { TR2-La calidad de mi trabajo mejora }\end{array}$ & $\begin{array}{l}\text { UA1-Los incentivos para estimular mis } \\
\text { capacidades y habilidades }\end{array}$ & \\
\hline $\begin{array}{l}\text { TP1-Estoy satisfecha/o con mi trabajo } \\
\text { en general }\end{array}$ & $\begin{array}{l}\text { UA2-El reconocimiento de mis activida- } \\
\text { des por parte de la institución y/o de } \\
\text { mis compañeros/as }\end{array}$ & \\
\hline $\begin{array}{l}\text { TP2-Estoy satisfecha/o con mi vida en } \\
\text { general }\end{array}$ & $\begin{array}{l}\text { UA3-La agilidad en la tramitación de so- } \\
\text { licitudes de proyectos... }\end{array}$ & \\
\hline
\end{tabular}

\section{ANÁLISIS Y RESULTADOS}

Del análisis descriptivo resultan las medias y las pruebas de normalidad (Tabla II). Estas últimas son positivas, por lo que se aplica el método de máxima verosimilitud (ML) al modelo estructural.

En cuanto a la adecuación muestral para la aplicación del ACP, una KMO de 0,753 y una prueba de esfericidad de Bartlett con Chi-cuadrado 7.732,892; 210 grados de libertad ( $d f$ ) y sig. 0 indican la idoneidad de su empleo.

\subsection{Análisis Factorial Exploratorio}

Los resultados del análisis exploratorio con rotación Varimax y método de Kaiser-Meyer-Oklin indican que la variable UE3 no tiene fuerza explicativa suficiente (peso<0,4), por lo que se excluye. Hecho esto, un segundo análisis revela 6 factores o constructos (ver Tabla III) que explican el 70,96\% de la variabilidad de los datos, lo que valida estos resultados (Hair, Anderson, Tatham y Black, 1995, p. 378).

A continuación y siguiendo a autores como Fuentes (1986), Steiger (1990) o Austin y Calde- rón(1996), se propone el modelo causal, cuyos constructos o dimensiones con sus correspondientes variables se exponen en la Tabla IV, y se valida: especificándolo, identificándolo, estimando sus parámetros y evaluándolo.

\subsection{Especificación e identificación del modelo y es-} timación de parámetros

Tras una primera estimación aparecen tres variables (TP2, UE1 y TP1) cuyo peso en la explicación de sus constructos no es significativo, por lo que son desechadas y se plantea el modelo de medida ajustado que aparece en la Figura 1.

En cuanto a su identificación, se puede afirmar que lo está, puesto que presenta 111 grados de libertad ( $d f$ y y es recursivo (Batista y Coenders, 2000). A su vez, las variables que explican cada constructo presentan correlaciones significativas y fuertes niveles de consistencia interna y fiabilidad (alphas de Cronbach $>0,8$ en todos los casos), de donde se infiere que la estimación de parámetros es adecuada (Nunnally, 1978). 
Tabla II. Resultados Análisis Descriptivo

\begin{tabular}{|cccc}
\hline & Media & Asimetría & Curtosis \\
\hline TF3 & 4,93 & $-0,418$ & $-0,422$ \\
\hline TF4 & 4,45 & $-0,112$ & $-0,891$ \\
\hline TF5 & 3,56 & 0,761 & $-0,046$ \\
\hline T13 & 5,02 & $-0,801$ & 0,095 \\
\hline T14 & 4,65 & $-0,567$ & $-0,588$ \\
\hline TI5 & 3,9 & $-0,107$ & $-1,104$ \\
\hline TR1 & 5,71 & $-1,257$ & 2,043 \\
\hline TR2 & 5,79 & $-1,279$ & 2,326 \\
\hline TP1 & 5,4 & $-1,029$ & 1,37 \\
\hline TP2 & 5,61 & $-1,103$ & 1,631 \\
\hline UF1 & 4,18 & $-0,254$ & $-0,877$ \\
\hline UF2 & 3,88 & $-0,055$ & $-0,824$ \\
\hline UF3 & 3,93 & $-0,081$ & $-0,811$ \\
\hline UE1 & 3,81 & $-0,072$ & $-0,88$ \\
\hline UE2 & 5,02 & $-0,661$ & $-0,337$ \\
\hline UE3 & 4,72 & $-0,551$ & $-0,528$ \\
\hline UA1 & 4,63 & $-0,544$ & $-0,507$ \\
\hline UA2 & 4,86 & $-0,647$ & $-0,441$ \\
\hline UA3 & 4,36 & $-0,281$ & $-0,999$ \\
\hline & & & \\
\hline
\end{tabular}

\subsection{Evaluación del modelo}

El modelo factorial confirmatorio permite explicar la correlación entre constructos, así como la asociación entre cada constructo y sus correspondientes variables observadas, mediante el cálculo de sus cargas factoriales (Tabla V).

En este caso, dichas cargas oscilan entre 0,7-0,9 $(>0,5 ;<1)$ y los errores no resultan significativos (Schumacker y Lomax, 2004), lo que valida el path-diagram propuesto y ofrece una imagen del alto grado explicativo de cada una de las variables respecto a su constructo (ver Figura 2).

Las correlaciones entre constructos varían en gran medida, registrándose tanto valores significativos (constructos relativos a la satisfacción del individuo) como inapreciables (constructos de infraestructuras universitarias con el resto de constructos). No ocurre lo mismo con el valor explicativo de los constructos fruto del ACP respecto al constructo comunicación de resultados de investigación (CRI), donde se observa que todos tienen una influencia directa semejante sobre él (ver ambos resultados en Tabla VI).
Tabla III. Resultados ACP

\begin{tabular}{|c|c|c|c|c|c|c|}
\hline & \multicolumn{6}{|c|}{ Componente } \\
\hline & 1 & 2 & 3 & 4 & 5 & 6 \\
\hline UF2 & ,879 & & & & & \\
\hline UF3 & ,876 & & & & & \\
\hline UF1 & ,850 & & & & & \\
\hline UE1 & ,529 & & & & & \\
\hline TP1 & ,520 & & & & & \\
\hline TI4 & & ,801 & & & & \\
\hline TF4 & & 797 & & & & \\
\hline TI3 & & 787 & & & & \\
\hline TF3 & & ,768 & & & & \\
\hline UA2 & & & ,839 & & & \\
\hline UA3 & & & , 817 & & & \\
\hline UA1 & & & ,774 & & & \\
\hline TR1 & & & & 902 & & \\
\hline TR2 & & & & ,895 & & \\
\hline TF5 & & & & & ,740 & \\
\hline TI5 & & & & & ,662 & \\
\hline TP2 & & & & & ,533 & \\
\hline UE2 & & & & & & 900 \\
\hline
\end{tabular}

En cuanto al ajuste del modelo, las medidas absolutas, incrementales y de parsimonia nos indican que este resulta aceptable y de calidad, y la probabilidad de que se mantenga el resultado analizando otra muestra es razonable.

\section{DISCUSIÓN Y CONCLUSIONES}

\subsection{Discusión}

Las variables con medias más altas corresponden (por este orden) a la incidencia en el rendimiento productivo de las vivencias de satisfacción laboral (TR1 y TR2), a la satisfacción general con la vida (TP2), a la satisfacción laboral general (TP1), a la intensidad de la satisfacción por la tarea investigadora (TI3) y a la satisfacción por el trabajo en espacios personales no universitarios (UE2). Las medias más bajas corresponden a la satisfacción de participar en congresos y seminarios (TF5 y TI5), a la satisfacción de trabajar en espacios de la organización compartidos (UE1), y a la provisión de recursos formativos, materiales e inmateriales por parte de la organización a los investigadores (UF2 y UF3), respectivamente. 
Tabla IV. Constructos propuestos y variables que los conforman

\begin{tabular}{|c|c|c|c|}
\hline \multicolumn{2}{|r|}{ Constructo } & \multicolumn{2}{|r|}{ Variable Explicativa } \\
\hline Código & Descripción & Código & Descripción \\
\hline \multirow{4}{*}{ SINV } & \multirow{4}{*}{$\begin{array}{l}\text { Satisfacción en tareas de preparación y } \\
\text { redacción (frecuencia e intensidad) }\end{array}$} & TF3 & $\begin{array}{c}\text { Frecuencia de la satisfacción .......preparando } \\
\text { alguna investigación }\end{array}$ \\
\hline & & TF4 & ...redactando material de investigación \\
\hline & & TI3 & $\begin{array}{c}\text { Intensidad de la satisfacción....... preparando } \\
\text { alguna investigación }\end{array}$ \\
\hline & & TI4 & ...redactando material de investigación \\
\hline \multirow{3}{*}{ REND } & \multirow{3}{*}{$\begin{array}{l}\text { Rendimiento productivo derivado de la } \\
\text { satisfacción laboral (percepción personal) }\end{array}$} & TR1 & $\begin{array}{l}\text { Aumento del rendimiento laboral debido a la } \\
\text { satisfacción }\end{array}$ \\
\hline & & & \\
\hline & & TR2 & $\begin{array}{l}\text { Aumento de la calidad del trabajo debido a } \\
\text { la satisfacción }\end{array}$ \\
\hline \multirow{3}{*}{ SPER } & \multirow{3}{*}{$\begin{array}{l}\text { Satisfacción en tareas de comunicación (frecuencia e } \\
\text { intensidad) y satisfacción vital global. }\end{array}$} & TF5 & $\begin{array}{l}\text { Frecuencia de la satisfacción participando } \\
\text { activamente en congresos, seminarios... }\end{array}$ \\
\hline & & T15 & $\begin{array}{l}\text { Intensidad de la satisfacción participando } \\
\text { activamente en congresos, seminarios... }\end{array}$ \\
\hline & & TP2 & Satisfacción vital general \\
\hline \multirow{5}{*}{ UFAC } & \multirow{5}{*}{$\begin{array}{c}\text { Satisfacción laboral potenciada con formación } \\
\text { y otros instrumentos por las organizaciones, y } \\
\text { satisfacción laboral global. }\end{array}$} & UF1 & $\begin{array}{l}\text { Satisfacción potenciada cuando la } \\
\text { organización... ...permite desarrollar } \\
\text { habilidades y plantear nuevos retos }\end{array}$ \\
\hline & & UF2 & ...provee de herramientas de formación \\
\hline & & UF3 & $\begin{array}{l}\text {...provee de instrumentos materiales e } \\
\text { inmateriales }\end{array}$ \\
\hline & & UE1 & $\begin{array}{c}\text { Satisfacción en espacios de la organización, } \\
\text { aunque sean compartidos }\end{array}$ \\
\hline & & TP1 & Satisfacción laboral general \\
\hline \multirow{3}{*}{ UFAV } & \multirow{3}{*}{$\begin{array}{l}\text { Satisfacción laboral potenciada por incentivos, } \\
\text { reconocimiento y agilidad burocrática }\end{array}$} & UA1 & $\begin{array}{l}\text { Satisfacción potenciada por... ....incentivos } \\
\text { para estimular capacidades y habilidades }\end{array}$ \\
\hline & & UA2 & $\begin{array}{l}\text {...reconocimiento de la institución y/o } \\
\text { compañeros/as }\end{array}$ \\
\hline & & UA3 & $\begin{array}{l}\text {....agilidad en la tramitación de solicitudes de } \\
\text { proyectos... }\end{array}$ \\
\hline UEP & $\begin{array}{l}\text { Satisfacción debida al trabajo en un espacio } \\
\text { personal no universitario. }\end{array}$ & UE2 & $\begin{array}{c}\text { Satisfacción en ambientes personales no } \\
\text { universitarios }\end{array}$ \\
\hline \multirow[b]{2}{*}{ CRI } & \multirow[b]{2}{*}{ Comunicación de Resultados de Investigación } & PIE & № proyectos innovación educativa \\
\hline & & PUBL & № publicaciones en revistas indexadas \\
\hline
\end{tabular}


Figura 1. Path-diagram (modelo de medida) propuesto

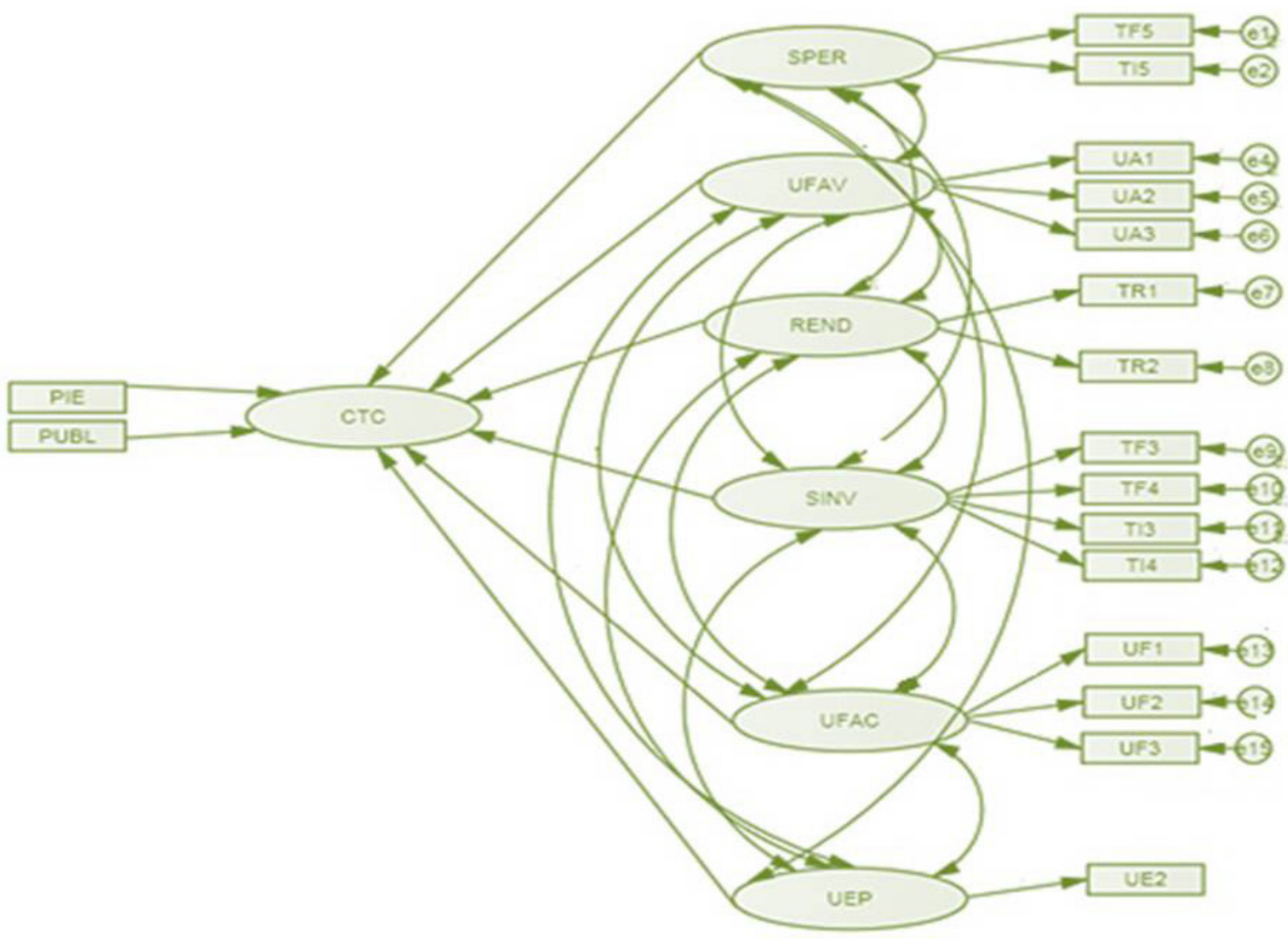

Figura 2. Modelo estructural estimado y sus ajustes

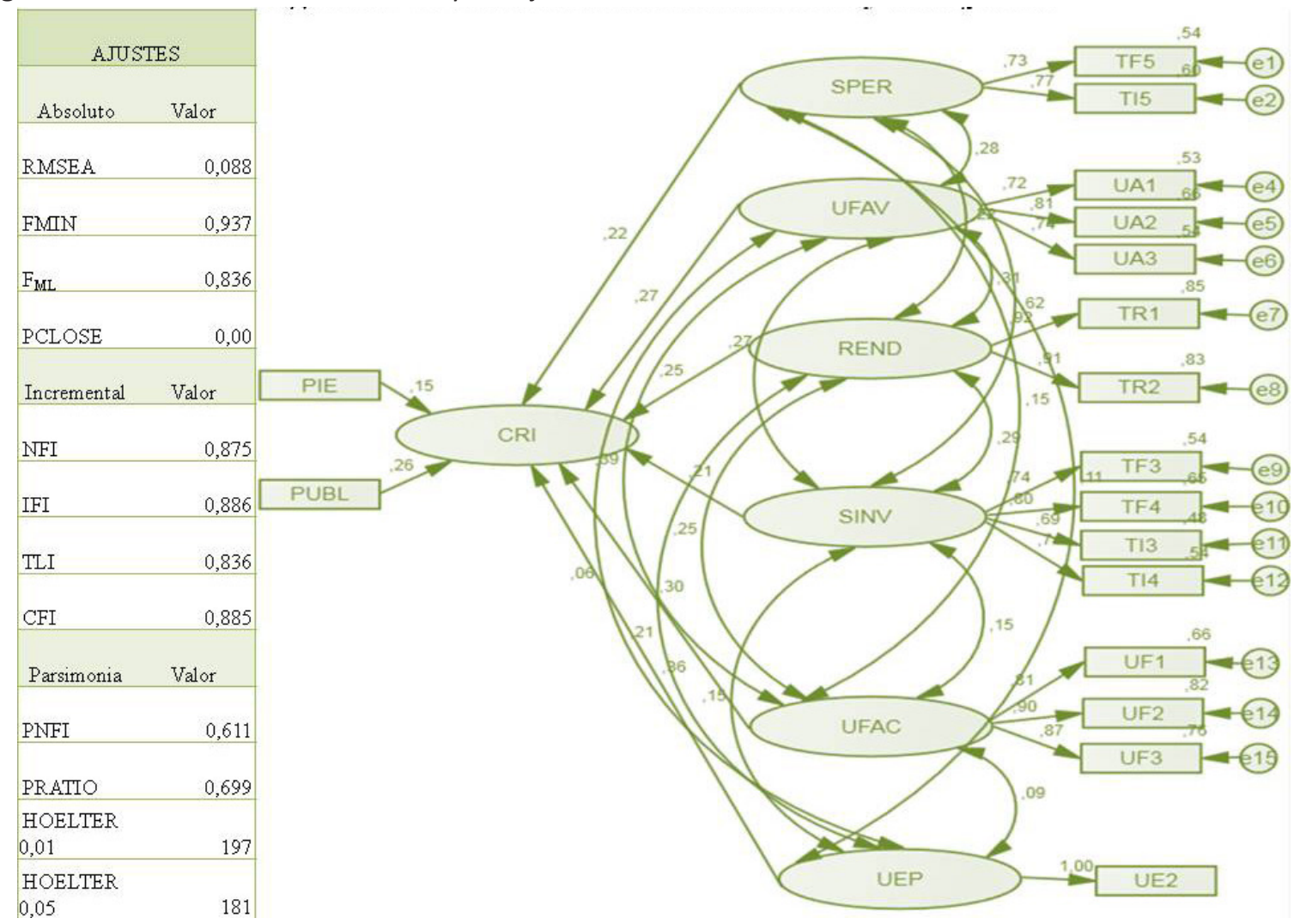


Tabla V. Pesos de las variables sobre sus constructos

\begin{tabular}{|cccc|}
\hline TF5 & $<---$ & SPER & 0,735 \\
\hline TI5 & $<---$ & SPER & 0,774 \\
\hline UA1 & $<---$ & UFAV & 0,725 \\
\hline UA2 & $<---$ & UFAV & 0,811 \\
\hline UA3 & $<---$ & UFAV & 0,738 \\
\hline TR1 & $<---$ & REND & 0,928 \\
\hline TR2 & $<---$ & REND & 0,9 \\
\hline TF3 & $<---$ & SINV & 0,738 \\
\hline TF4 & $<---$ & SINV & 0,804 \\
\hline TI3 & $<---$ & SINV & 0,689 \\
\hline TI4 & $<---$ & SINV & 0,735 \\
\hline UF1 & $<---$ & UFAC & 0,81 \\
\hline UF2 & $<---$ & UFAC & 0,904 \\
\hline UF3 & $<---$ & UFAC & 0,874 \\
\hline & & & \\
\hline
\end{tabular}

A la hora de explicar los constructos, si observamos los resultados del ACP corregidos con el modelo de medida SEM, las variables con más fuerza explicativa son las del constructo rendimiento (REND) tanto en cantidad (TR1, con un peso de 0,928) como en calidad (TR2, con un peso de 0,9 ), siguiéndole con pesos entre 0,8 y 0,9 las variables explicativas de la satisfacción laboral potenciada por las organizaciones (en orden descendente, UF2, UF3, UF1), absorbiendo a la satisfacción laboral global (TP1). Las variables explicativas de los constructos relativos a la satisfacción en sí oscilan entre 0,7 y 0,8, cabiendo destacar que la satisfacción con la investigación en sí (SINV) engloba diferentes niveles de satisfacción procedentes de la preparación y redacción de resultados de investigación (en orden descendente, TF4, TF3, TI4, TI3), mientras que la satisfacción personal (SPER) proviene de la obtenida en tareas de participación en congresos (TF5, TI5), quedando la satisfacción vital global (TP2) englobada en ellas.

Las relaciones causales entre los constructos planteados son significativas tanto en el caso de correlaciones entre ellos como de regresión entre resultados del ACP y el constructo planteado comunicación de resultados de la investigación (CRI), por lo que han de ser tomadas en consideración.
Tabla VI. Valor de las relaciones causales entre constructos

\begin{tabular}{|cccc}
\hline \multicolumn{4}{c}{$\begin{array}{c}\text { Correlaciones entre constructos que inciden en la } \\
\text { Comunicación de Resultados de Investigación }\end{array}$} \\
\hline SPER & $<-->$ & UFAV & 0,282 \\
\hline UFAV & $<-->$ & REND & 0,312 \\
\hline REND & $<-->$ & SINV & 0,288 \\
\hline SPER & $<-->$ & REND & 0,225 \\
\hline SPER & $<-->$ & SINV & 0,624 \\
\hline UFAV & $<-->$ & SINV & 0,265 \\
\hline SINV & $<-->$ & UFAC & 0,151 \\
\hline SPER & $<-->$ & UFAC & 0,146 \\
\hline UFAV & $<-->$ & UFAC & 0,392 \\
\hline REND & $<-->$ & UFAC & 0,253 \\
\hline UEP & $<-->$ & UFAC & 0,089 \\
\hline UEP & $<-->$ & SINV & 0,15 \\
\hline UEP & $<-->$ & REND & 0,207 \\
\hline UEP & $<-->$ & UFAV & 0,064 \\
\hline UEP & $<-->$ & SPER & 0,107 \\
\hline Varianza de la Comunicación de Resultados de \\
\hline Investigación explicada por el resto de los constructos \\
\hline CRI & $<---$ & SPER & 0,218 \\
\hline CRI & $<---$ & UFAV & 0,269 \\
\hline CRI & $<---$ & REND & 0,25 \\
\hline CRI & $<---$ & SINV & 0,212 \\
\hline CRI & $<---$ & UFAC & 0,3 \\
\hline CRI & $<--$ & UEP & 0,356 \\
\hline
\end{tabular}

Las correlaciones son altas entre los constructos centrales de satisfacción (SINV y SPER, o satisfacción obtenida en tareas de preparación y redacción, en comunicación, y satisfacción global), y moderadas entre ambos y el constructo de rendimiento debido a la satisfacción (REND, incidiendo las anteriores variables centrales de satisfacción tanto en rendimiento cuantitativo TR1 como en calidad TR2).

Finalmente, la comunicación de resultados de investigación (CRI) se determina más por los constructos facilitadores (por UEP, UFAC y UFAV, respectivamente, con pesos de regresión en torno a 0,3) que por los de satisfacción (SINV, REND y SPER, con pesos de regresión en torno a 0,2 ). Resalta que el constructo y variable más influyente sea la satisfacción debida al trabajo en ambientes propios no universitarios (cons- 
tructo UEP con única variable UE2), y más cuando el efecto de la satisfacción lograda en espacios universitarios (UE1 y UE3) queda absorbido por el de la lograda mediante provisión de otras condiciones organizacionales (UF) a la hora de explicar los constructos. El siguiente constructo con fuerza para explicar la comunicación de resultados de investigación (CRI) es la satisfacción laboral potenciada con formación y otros instrumentos por las organizaciones, y satisfacción laboral global (UFAC), pero teniendo mucho menor peso la potenciada con incentivos, reconocimiento y agilidad burocrática (UFAV). En cuarto, quinto y último lugar se hacen notar los constructos de satisfacción.

\subsection{Conclusiones}

Según el estudio, cuando el personal docente e investigador de las universidades reporta sobre la esencia y efectos de su satisfacción laboral, enfatiza el rendimiento productivo derivado de ella, su satisfacción general con la vida, la satisfacción lograda por las tareas investigadoras en sí mismas y el poder realizarlas en espacios propios, no universitarios, suficientemente equipados. Esto último puede resultar sorprendente en ciencias experimentales y tecnológicas que requieren espacios sofisticados, pero debe obviamente entenderse como una referencia a la preparación y al procesamiento de la información derivadas de las actividades en tales ámbitos (es decir, preparar los proyectos de experimentos y actos físicos, y redactar finalmente los resultados para su publicación).

A su vez, dicho personal percibe que es relativamente moderada la influencia que sobre su satisfacción laboral ejerce la universidad al proveer de recursos, de una gestión facilitadora y de espacios laborales. Ahora bien, si analizamos este escenario en función de conocer los determinantes de la comunicación de resultados de investigación, el relato se altera sustancialmente hasta casi invertirse.

Esto es así porque dichas variables no son las que inciden de forma más significativa en la comunicación de resultados de investigación, más bien al contrario. De ahí que, la alteración fundamental de los énfasis se da a la hora de comprender, precisamente, los determinantes fundamentales de la comunicación de resultados de investigación. Ésta se determina más por la satisfacción potenciada por las infraestructuras y apoyos universitarios que por los disfrutes espontáneos del investigador en sus diferentes tareas. Ahora bien, no todo apoyo por parte de la organización tiene el mismo efecto. Lo que más ayuda a aumentar la comunicación de resultados de investigación es el trabajar en espacios propios no universitarios que, no obstante, cuenten con suficiente equipamiento. El efecto de la satisfacción lograda en espacios universitarios es mucho menos relevante, y queda absorbido por el de la satisfacción derivada de otros apoyos organizacionales. Entre ellos resaltan las posibilidades de formación u otros instrumentos materiales e inmateriales, pero con menor peso los incentivos, el reconocimiento y la agilidad burocrática.

Los resultados son acordes con la gestión basada en la motivación personal y con el de 'flow' o satisfacción por las tareas en sí mismas planteados como marco introductorio, y resaltan además las características de productividad laboral del trabajador del conocimiento (Drucker, 1999; Nonaka y Takeuchi, 1995; Smith, Collins y Clark, 2005), más centrada en aspectos referentes a la adquisición y transmisión constante de conocimientos, integración de las TIC's como herramientas facilitadoras, calidad y aportación de valor que prevalecen sobre la cantidad, integración de ambientes personales, sociales y corporativos, e integración del ocio en las propias zonas de trabajo. La productividad de las universidades puede estudiarse de manera cuantitativa en diferentes indicadores (Pérez García y Serrano Martínez, 2012), pero mientras no pase este filtro de las percepciones y motivaciones personales parece arduo que pueda alcanzar rendimientos sostenibles.

En suma, la comunicación de resultados de investigación de una universidad, entendida fundamentalmente como número de artículos indexados, pero ampliando el campo explicativo con la investigación práctica de los Programas de Innovación Educativa, se ve potenciada y explicada (en orden descendente) por: la posibilidad de trabajar en ambientes personales no universitarios pero bien equipados; la posibilidad de desarrollar habilidades y plantearse nuevos retos, así como de formarse y de proveerse de otros instrumentos materiales o inmateriales; los incentivos, el reconocimiento y la agilidad burocrática; la percepción personal del rendimiento productivo derivado de la satisfacción laboral; la satisfacción personal en tareas de comunicación en congresos y otros escenarios; y la satisfacción en tareas de preparación y redacción de material de investigación.

Las implicaciones respecto a las políticas a tomar por parte de las organizaciones para mejorar la comunicación de sus investigaciones, y su maniobrabilidad, parecen claras: resultará más efectivo dar libertad para el trabajo en espacios propios no universitarios, procurando que estén bien equipados con equipos informáticos fácilmente transportables e intranets ágiles y po- 
tentes, y no tan efectivo invertir en infraestructuras en los espacios de la propia universidad para este tipo de menesteres (sin que lo primero excluya lo último). 0 , como vía intermedia, resultará más efectivo invertir en hacer 'familiares' o 'propias' sus infraestructuras y espacios que en su aumento o modernización. Sobre esta base, debe mejorarse el marco inmaterial de apoyos a la investigación. Otros factores, como la percepción del rendimiento derivada de la satisfacción en labores de investigación y dicha satisfacción en sí, quedan más lejos del alcance de la gestión universitaria y requerirán medidas más imaginativas, pero deberán atenderse igualmente. De facto, en esta tríada de apoyo a la investigación formada por espacios y apoyos materiales, apoyos inmateriales y atención a la satisfacción, esta última parece sobresalir como línea a investigar y aspecto organizacional a profundizar, dado que suele ser el más descuidado de los tres ejes.

Como limitaciones principales de este trabajo cabe destacar que únicamente se han utilizado variables de satisfacción y su relación con la comunicación de resultados en artículos indexados, dejando aparte variables que parecen incidir sobre la comunicación científica como son los incentivos de promoción económica (sexenios de investigación) y académica (acreditaciones), (Fernández-Quijada y Masip, 2013); o el prestigio y líneas estratégicas de la Universidad. Así mismo, el estudio se circunscribe a una única universidad, la UPV/EHU, por lo que puede resultar arriesgado extrapolar los resultados obtenidos a otras universidades públicas o privadas; nacionales o europeas.

Dada la importancia personal y organizacional de la comunicación científica y de la satisfacción de los miembros de la organización, tanto un estudio basado en variables específicas de comunicación científica como la ampliación del presente estudio a otras universidades públicas del Estado serán objeto de investigaciones futuras así como la comparación de resultados con posibles estudios similares de universidades europeas.

\section{BIBLIOGRAFÍA}

Aitken, N. D. (1982). College student performance, satisfaction and retention: Specification and estimation of a structural model. The Journal of Higher Education, pp. 32-50. http://dx.doi. org/10.2307/1981537

APA. (2010). Publication manual of the american psychological association 6th edition. Washington, DC: American Psychological Association

Arbuckle, J. L. (2006). Amos ${ }^{T M} 7.0$ user's $^{2}$ guide Amos Development Corporation

Austin, J. y Calderon, R. (1996). Theorical and technical contributions to structural equations modelling: An updated bibliography. Structural Equation Modeling, 3, 2, pp. 101-125. http://dx.doi. org/10.1080/10705519609540036

Barjak, F. (2006). The role of the internet in informal scholarly communication. Journal of the American Society for Information Science and Technology, 57, 10, pp. 1350-1367. http://dx.doi. org/10.1002/asi.20454

Batista, J. M. y Coenders, G. (2000). Modelos de ecuaciones estructurales (6a ed.). Madrid: La Muralla

Beall, J. (2014). The future of scholarly communication. Portal : Libraries and the Academy, 14(1), 121-122. http://dx.doi. org/10.1353/pla.2013.0042
Bisquerra Alzina, R. (1989). Introducción conceptual al análisis multivariable. Un enfoque informático con los paquetes SPSS- $X$, BMDP, LISREL y SPAD. Barcelona: PPU

Björk, B.-C. (2007). A model of scientifitc communication of a global distributed information system. 2007 IATUL Proceedings. Disponible en http:// docs.lib.purdue.edu/cgi/viewcontent. cgi article $=1797 \&$ context=iatul

Burke, R. J., Koyuncu, M. y Fiksenbaum, L. (2008). Workaholism, work and extra-work satisfactions and psychological well-being among professors in turkey. Cross Cultural Management, 15, 4, pp. 353-366. http://dx.doi. org/10.1108/13527600810914148

Bussing, A., Bissels, T., Fuchs, V.y Perrar, K. M. (1999). A dynamic model of work satisfaction: Qualitative approaches. Human Relations, 52, 8, pp. 999-1028. http:// dx.doi.org/10.1023/A:1016979523846

Castelli, D., Manghi, P. y Thanos, C. (2013). A vision towards scientific communication infrastructures. International Journal on Digital Libraries, 13, 3-4, pp. 155-169. http://dx.doi.org/10.1007/ s00799-013-0106-7

Cohen, J. (1996). Computer mediated communication and publication productivity among faculty. Internet Re- search, 6, 2/3, pp. 41-63. http://dx.doi. org/10.1108/10662249610127328

Conklin, M. H. y Desselle, S. P. (2007). Development of a multidimensional scale to measure work satisfaction among pharmacy faculty members. American Journal of Pharmaceutical Education, 71, 4, pp. 1-61. http://dx.doi.org/10.5688/ aj710461

Csíkszentmihályi, M. (1975). Beyond boredom and anxiety: The experience of play in work and games. London: JosseyBass.

Csíkszentmihályi, M. (1990). Flow: The psychology of optimal experience. New York: Harper Collins.

de León, P. C. (2002). La innovación educativa. Madrid: Akal

Diener, E. (2009). Subjective well-being. En Diener, E. The science of well-being. Springer Netherlands, pp. 11-58. http://dx.doi. org/10.1007/978-90-481-2350-6_2

Drucker, P. F. (1993). Post capitalist society. Oxford: Harper Collins.

http://dx.doi.org/10.1016/B978-0-75060921-0.50009-4

Drucker, P. F. (1999). Knowledge-worker productivity: The biggest challenge. Harvard Business Review, 41, 2, pp. 72-96. http://dx.doi.org/10.2307/41165987 
Eid, M. y Larsen, R. J. (eds.). (2008). The science of subjective well-being. Guilford Press

Elçi, M. y Alpkan, L. (2009). The impact of perceived organizational ethical climate on work satisfaction. Journal of Business Ethics, 84, 3, pp. 297-311. http://dx.doi. org/10.1007/s10551-008-9709-0

Falkenburg, K. y Schyns, B. (2007). Work satisfaction, organizational commitment and withdrawal behaviours. Management Research News, 30, 10, pp. 708-723. http://dx.doi. org/10.1108/01409170710823430

Fernández-Quijada, D. y Masip, P. (2013). Tres décadas de investigación española en comunicación: hacia la mayoría de edad (three decades of spanish communication research: Towards legal age). Comunicar, 41, XXI, 15-24.

Fuentes, A. (1986). Los modelos causales en la investigación del rendimiento académico. Revista de Investigación Educativa RIE, 4, 7, pp. 35-48.

García, C. M. (dir.) (1996). Innovación educativa, asesoramiento y desarrollo profesional Madrid: Centro de Publicaciones. Ministerio de Educación y Ciencia

García, C. M., Ruiz, C. M. y Noche, B. G. (2010). Innovación educativa en España desde el punto de vista de sus protagonistas. Profesorado: Revista de Curriculum y Formación del Profesorado, 14, 1, pp. 111-134.

Hackman, J. R. y Oldham, G. R. (2005). How job characteristics theory happened. In Smith K. G. y Hitt M. A. (eds.). The oxford handbook of management theory: The process of theory development. Oxford, UK: Oxford University Press, pp. 151-170.

Hair, J. F., Anderson, R. E., Tatham, R. y Black, W. (1995). Multivariate data analysis. Englewood Cliffs, NJ: Prentice Hall

Harley, D. (2013). Scholarly communication: Cultural contexts, evolving models. Science, 342, 6154, pp. 80-82. http:// dx.doi.org/10.1126/science.1243622

Herrera, T. P. (2010). La práctica de la innovación educativa. Comunicar, 17, 34, p. 227

Herzberg, F. (1968). One more time: How do you motivate employees. Harvard Business Review, 46, 1, pp. 53-62.
House, E. R. (1988). Tres perspectivas de la innovación educativa: Tecnología, política y cultura. Revista de Educación, 286, pp. 5-34.

Jackson, S. A. (1996). Toward a conceptual understanding of the flow experience in elite athletes. Research Quarterly for Exercise and Sport, 67, 1, pp. 76-90. http://dx.doi.org/10.1080/02701367.1 996.10607928

Jackson, S. A. y Eklund, R. C. (2002). Assessing flow in physical activity: The flow-state scale-2 and dispositional flow scale-2. Journal of Sport \& Exercise Psychology, 24, 2, pp. 133-150. http:// dx.doi.org/10.1123/jsep.24.2.133

Jöreskorg, K. J. (1979). A general approach to confirmatory maximum likelhood factor analysis with addendum. En Jöreskorg, K. J., Sörbom, D. y Magidson, J. (eds.). Advances in factor analysis and structural equation models. Cambridge: Abt Books.

Judge, T. A., Thoresen, C. J., Bono, J. E. Y Patton, G. K. (2001). The job satisfaction-job performance relationship: A qualitative and quantitative review. Psychological Bulletin, 127, 3, 376407. http://dx.doi.org/10.1037/00332909.127.3.376

Kahneman, D. y Krueger, A. B. (2006). Developments in the measurement of subjective well-being. The Journal of Economic Perspectives, 20, 1, pp. 3-24. http://dx.doi. org/10.1257/089533006776526030

Kling, R. y McKim, G. (2000). Not just a matter of time: Field differences and the shaping of electronic media in supporting scientific communication. Journal of the American Society for Information Science, 51, 14, pp. 13061320. http://dx.doi.org/10.1002/1097$4571(2000) 9999: 9999<:$ : AID ASI1047>3.0.CO;2-T

Koyuncu, M., Burke, R. J. y Fiksenbaum, L. (2006). Work experience and satisfaction of male and female professors in turkey: Signs of progress? Equal Opportunities International, 25, 1, pp. 38-47. http://dx.doi. org/10.1108/02610150610645959

Maharaj, I. y Schlechter, A. F. (2007). Meaning in life and meaning of work: Relationships with organisational citizenship behaviour, commitment and job satisfaction. Management Dynamics, 16, 3, pp. 24-41.
Mann, F. y Likert, R. (1952). The need for research on the communication of research results. Human Organization, 11, 4, pp. 15-19. http://dx.doi.org/10.17730/ humo.11.4.k6p20p687j667144

Mayo, E. (1933). The human problems of an industrial civilization. New York: Macmillan

McGregor, D. (1957). The human side of enterprise. Cambridge, Mass: McGraw-Hill.

Morris, S. (1998). Learned journals and the communication of research. Learned Publishing, 11, 4, pp. 253-258. http://dx.doi. org/10.1087/09531519850146175

Nakamura, J. y Csíkszentmihályi, M. (2012). Flow theory and research. En: Lopez, S. J. y Snyder, C. R. (eds.). The Oxford handbook of positive psychology. Oxford, New York: Oxford University Press, pp. 195-205.

National Science Board (US). (2014). Science and engineering indicators 2014. Washington, DC: Government Printing Office.

Neuman, W. R., Davidson, R., Joo, S., Park, Y. J. y Williams, A. E. (2008). The seven deadly sins of communication research. Journal of Communication, 58, 2, pp. 220-237. http://dx.doi.org/10.1111/ j.1460-2466.2008.00382.x

Nonaka, I. y Takeuchi, H. (1995). The knowledge-creating company. New York, Oxford: Oxford University Press.

Nunnally, J. C. (1978). Psychometric theory ( 2 a ed.). New York: McGraw-Hill.

Odlyzko, A. (2002). The rapid evolution of scholarly communication. Learned Publishing, 15, 1, pp. 7-19. http://dx.doi. org/10.1087/095315102753303634

Ouchi, W. G. (1981). Theory Z: How American business can meet the Japanese challenge. Reading, Mass.: Addison-Wesley.

Pearson, L. C. y Moomaw, W. (2005). The relationship between teacher autonomy and stress, work satisfaction, empowerment, and professionalism. Educational Research Quarterly, 29, 1, pp. 37-53.

Pérez García, F. y Serrano Martínez, L. (dirs.) (2012). Universidad, universitarios $y$ productividad en España. Bilbao: Fundación BBVA.

Peters, T. J. y Waterman, R. H. (1983). En busca de la excelencia: Lecciones de las empresas mejor gestionadas de Estados unidos. Barcelona: Folio. 
Pfeffer, J. (1994). Competitive advantage through people: Unleashing the power of the work force. Boston, Mass.: Harvard Business School Press.

Phillips, L. J. (2011). Analysing the dialogic turn in the communication of research-based knowledge: An exploration of the tensions in collaborative research. Public Understanding of Science, 20, 1, pp. 80-100. http://dx.doi. org/10.1177/0963662509340092

Quintanal Díaz, J. Q. (2010). Sobre: Paredes, J. y de la Herrán, A. (coords.) (2009). La práctica de la innovación educativa. Educación XX1: Revista de la Facultad de Educación, 13, 1, pp. 223-224.

Røssberg, J. I., Eiring, $\varnothing$. y Friis, S. (2004). Work environment and job satisfaction. Social Psychiatry and Psychiatric Epidemiology, 39, 7, pp. 576-580. http:// dx.doi.org/10.1007/s00127-004-0791-z

Ruiz, M. A., Pardo, A. y San Martín, R. (2010). Modelos de ecuaciones estructurales. Papeles del Psicólogo, 31, 1, pp. 34-45.

Rupp-Serrano, K. y Antell, K. (2013). The future of scholarly communication. Reference \& User Services Quarterly, 53, 2, 192-193. http://dx.doi.org/10.5860/ rusq. $53 \mathrm{n} 2.192 \mathrm{c}$
Saratxaga, K. (2007). Un nuevo estilo de relaciones: Para el cambio organizacional pendiente. Madrid: Prentice Hall.

Schumacker, R. E. y Lomax, R. G. (2004). A beginner's guide to structural equation modeling. Mahwah: Lawrence Erlbaum Societies.

Senge, P., Roberts, C., Ross, R. B., Smith, B. J. y Kleiner, A. (1995). La quinta disciplina en la práctica. Estrategias y herramientas para construir la organización abierta al aprendizaje. Barcelona: Granica.

Shaw, W. M. Jr. (1981). Information theory and scientific communication. Scientometrics, 3, 3, pp. 235-249. http://dx.doi. org/10.1007/BF02101668

Shorley, D. y Jubb, M. (2013). The future of scholarly communication. London: Facet.

Smith, K. G., Collins, C. J. y Clark, K. D. (2005). Existing knowledge, knowledge creation capability, and the rate of new product introduction in high-technology firms. Academy of Management Journal, 48, 2, pp. 346-357. http://dx.doi. org/10.5465/AMJ.2005.16928421

Smith, M. (2009). Gender, pay and work satisfaction at a UK university. Gender, Work and Organization, 16, 5, pp. 621641. http://dx.doi.org/10.1111/j.14680432.2008.00403.x
Solomon, D. J. (2013). Digital distribution of academic journals and its impact on scholarly communication: Looking back after 20 years. Journal of Academic Librarianship, 39, 1, pp. 2328. http://dx.doi.org/10.1016/j.acalib.2012.10.001

Spence Laschinger, H. K., Finegan, J. E., Shamian, J. y Wilk, P. (2004). A longitudinal analysis of the impact of workplace empowerment on work satisfaction. Journal of Organizational Behavior, 25, 4, pp. 527-545. http:// dx.doi.org/10.1002/job. 256

Steiger, J. H. (1990). Structural model evaluation and modification: An interval estimation approach. Multivariate Behavioral Research, 25, 2, pp. 173-180. http://dx.doi.org/10.1207/ s15327906mbr2502 4

Tapscott, D. y Williams, A. D. (2007). Wikinomics: La nueva economía de las multitudes inteligentes. Barcelona: Paidós.

\section{Recursos de Internet}

World University Rankings 2011-12 methodology. [En línea]. [Fecha de consulta: 21 mayo 2014] Disponible en https://www. timeshighereducation.com/world-university-rankings-2011-12-methodology 